\title{
A case report on the long-term survival of a patient with HER2-positive metastatic gastric adenocarcinoma and a short review of the current literature
}

\author{
Hannah Christina Puhr · Aysegül Ilhan-Mutlu (D)
}

Received: 5 August 2020 / Accepted: 1 September 2020 / Published online: 30 September 2020

(C) The Author(s) 2020

\begin{abstract}
Summary The prognosis of patients with metastatic gastroesophageal cancer remains poor despite numerous promising clinical trials, and the clinical benefit of systemic therapies is under critical review. This case report of a patient with human epidermal growth factor receptor 2 (HER2)-positive metastatic gastric adenocarcinoma is an impulse for the importance of individual decision making and molecular guided treatment options.
\end{abstract}

Keywords Gastric cancer · Upper GI cancer - HER2 • Trastuzumab · Ramucirumab

\section{Case presentation}

A 70-year-old man presented with upper abdominal pain at the general practitioner in January of 2016 . Medical history showed no prior illness or surgeries and, thus, no previous medication. In the physical examination, a reduced nutritional status with $65 \mathrm{~kg}$ at a height of $1.82 \mathrm{~m}$ became evident, but no other physical abnormalities could be detected. No dyspepsia or dysphagia was reported. An endoscopy showed a tumor in the antrum of the stomach with suspected covered perforation of the gastric wall.

The patient was then transferred to our hospital, and despite not being able to confirm the suspected perforation in a computed tomography, the patient

H. C. Puhr, MD · A. Ilhan-Mutlu, MD PhD ( $\bowtie)$

Department of Medicine I, Division of Oncology, Medical University of Vienna, Vienna, Austria

Comprehensive Cancer Centre Vienna, Upper

Gastrointestinal Tumors Unit, Medical University of Vienna,

Waehringer Guertel 18-20, 1090 Vienna, Austria

aysegul.ilhan@meduniwien.ac.at

H. C. Puhr, MD

hannah.puhr@meduniwien.ac.at was operated on shortly thereafter. A subtotal gastrectomy was performed. The histological report showed an already advanced HER2 (human epidermal growth factor receptor 2)-positive (ICH 3+) adenocarcinoma with lymph node as well as omental metastases (G3, pT3, L1, V0, Pn1, pN2a, pM1, R0). There was no visible residual disease. Adjuvant chemotherapy after the surgery was declined by the patient.

In September 2016, the patient presented with multiple liver and abdominal lymph node metastases and by then agreed to undergo chemotherapy. Six cycles of chemotherapy following the ToGA regimen (trastuzumab, cisplatin, capecitabine) were administered and partial remission was achieved. The patient experienced several chemotherapy-induced toxicities including infections, low white blood count, and acral erythema. Thus, chemotherapy was terminated after 6 cycles and a maintenance therapy with trastuzumab was established beginning in March 2017. This therapy was continued until October 2018, when restaging showed progression of lymph node metastases. Radiation therapy on the local lymph nodes together with oral capecitabine were administered between October and March 2019. Trastuzumab maintenance therapy was continued.

The next restaging in March 2019 showed a progressive disease with new pulmonal lesions and carcinomatosis peritonei. So far he had received 39 cycles of trastuzumab and it was surmised that the tumor might had lost its HER2-positivity. Furthermore, the patient presented with an ileus in April 2019, which had to be managed surgically with a colostomy. Due to this progress, the patient received second-line therapy with ramucirumab plus paclitaxel from April until October 2019, which induced a partial response after 6 cycles. Due to good response, maintenance therapy with ramucirumab was established in October 2019 until December 2019. 


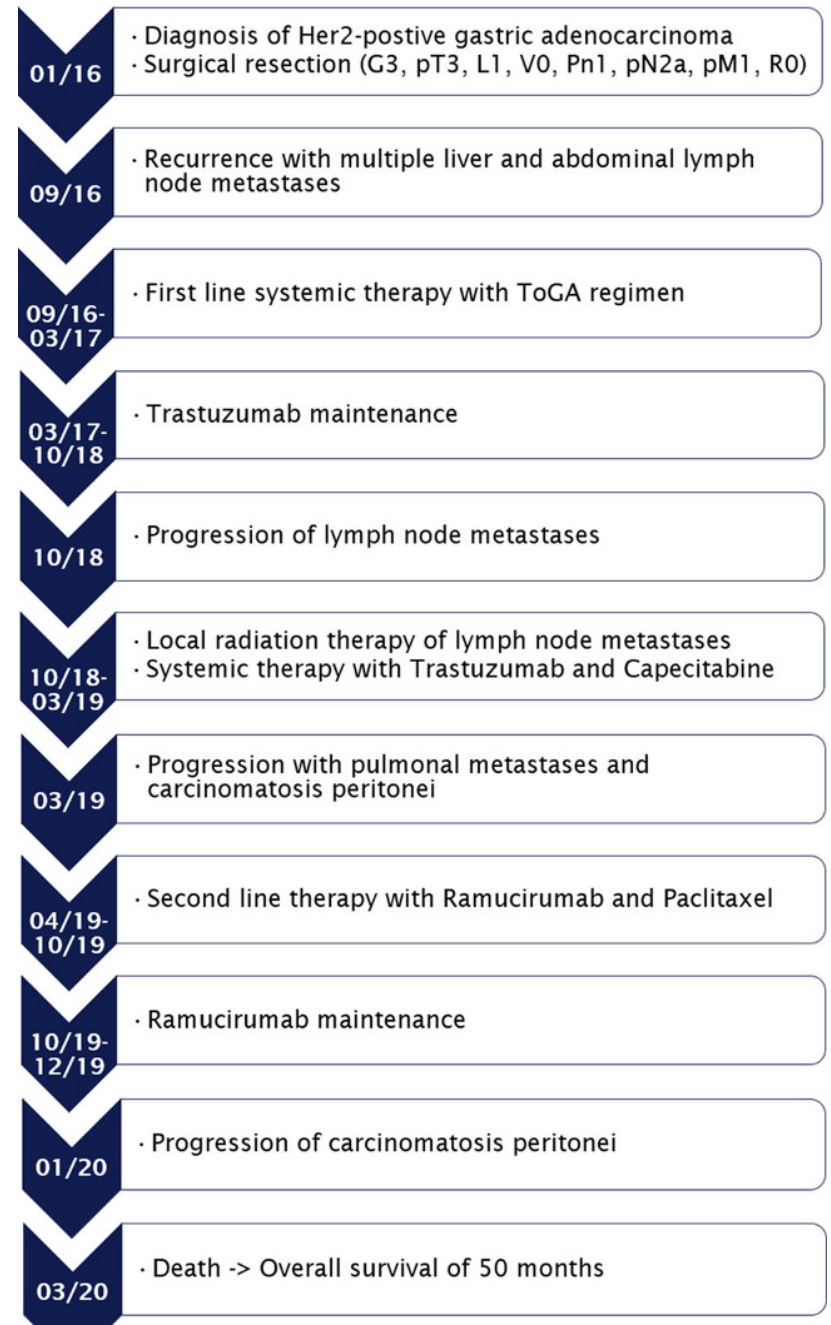

Fig. 1 Timeline of disease evolution

The patient wished to reverse the colostomy in January 2020. However, during the surgery a massive Carcinosis peritonei was detected and biopsied. Unfortunately no HER2 analysis was performed. The biopsy was microsatellite stable. Due to the progression of the peritoneal carcinomatosis, maintenance treatment with ramucirumab was discontinued after 2 months. A standard third-line treatment with trifluridin and tipiracil was discussed with the patient. After surgery the status of the patient deteriorated quickly and he died in March 2020 in the palliative care unit due to rapid tumor progression. The overall survival (OS) was 50 months (Fig. 1).

\section{Critical discussion and review of the literature}

This case report of a patient with initially metastatic, HER2-positive gastric adenocarcinoma attends several important steps concerning treatment options.

\section{Surgery}

First, the patient received a subtotal gastrectomy due to the suspected perforation and the lack of evidence of advanced disease in the computed tomography. Even though it was evident once the surgery began that he was in a locally advanced state, the gastrectomy was performed to treat the suspected perforation. Without the suspected perforation the treatment of choice would have been a perioperative chemotherapy regimen, with a neoadjuvant regimen of FLOT (leucovarin, 5-fluorouracil, oxaliplatin, and docetaxel). This treatment regimen represents a quite novel treatment option, which showed promising results in a first-line perioperative setting [1]. This regimen can be considered as first-line therapy for patients with an excellent performance status. Furthermore, there were several trials to evaluate the role of HER2-targeted therapies in a first-line resectable setting of gastroesophageal adenocarcinoma; however, so far there have been no practice-changing results $[2,3]$.

\section{Perioperative systemic therapy}

The patient refused even adjuvant chemotherapy and only a few months later developed rapid recurrence with liver and lymph node metastases. This underlines the importance of adjuvant treatment particularly in advanced settings [4].

\section{Palliative systemic therapy-first line}

Due to the palliative state of the HER2-positive metastatic, gastric adenocarcinoma, a treatment according to the ToGA trial was administered [5]. Although this regimen provides a widely prolonged survival, the toxicities often lead to severe adverse events. Thus, several trials try to exchange cisplatin with oxaliplatin, seen as it has a more favorable toxicity profile [6]. However, so far no new treatment options for this setting are available. Also, other treatment strategies for HER2-positive gastric cancer have so far failed to improve the overall survival (OS) [7, 8].

After 6 cycles of therapy partial remission and an excellent performance score could be achieved; thus, trastuzumab maintenance therapy was established according to recently published observations [9-11].

\section{Radiation therapy}

This maintenance was continued for 7 months, until there was a minor progression in lymph nodes metastases; however, stable disease concerning the liver metastases was evident. Thus, it was decided to treat the localized lymph node metastasis with radiotherapy and to expand the maintenance therapy with capecitabine. Radiation therapy of localized lymph 
node metastases is known to be effective as palliative treatment and to prolong OS [12, 13].

\section{Palliative systemic therapy-second line}

After 39 cycles of trastuzumab treatment, there was a progression with expansion of known liver and lymph node metastases as well as development of pulmonal metastases. Although trastuzumab proved to be very effective in our patient, we decided not to continue this treatment beyond progression. This decision was based on the results of a phase II study comparing the combination of paclitaxel beyond progression with trastuzumab versus paclitaxel alone, which showed that there was no benefit of combination therapy concerning OS [14]. Furthermore, other trials that targeted HER2 in a second-line strategy also failed to improve OS $[15,16]$. It is surmised that this effect might be due to the loss of HER2 after first-line treatment [17].

Thus, we decided to further manage this patient as a HER2-negative case and applied the standard second-line chemotherapy with ramucirumab and paclitaxel [18]. A recent subgroup analysis of the initial trial also demonstrated efficacy benefits of this regimen after trastuzumab-based treatment [19]. However, the European Society of Medical Oncology-Magnitude of Clinical Benefit Scale (ESMO-MCBS), a score which indicates the substantial magnitude of clinical benefit of particular novel treatment options in oncology, is only 2 for ramucirumab and paclitaxel [20]. Thus, the clinical benefit for the patient might be minimal and that is why this regimen is critically viewed and widely discussed as a second-line option. However, our patient had a subjective benefit and good response throughout this therapy.

\section{Palliative systemic therapy-third line}

As a third line, we discussed to establish a therapy with trifluridin and tipiracil. This treatment option was published in 2018 and showed promising results on OS and quality of life when compared to placebo [21, 22]. Unfortunately, our patient died before this therapy regimen could be administered. The rapid deterioration after the surgery to reverse the colostomy was associated with rapid tumor progression. The cause for this rapid progression is not evident and might be due to the discontinuation of the antiangiogenic drug ramucirumab, the surgery itself [23], late knowledge of carcinomatosis due to low detection rates in CT scans [24] as well as other various unknown reasons.

\section{Survival and prognosis}

Despite the success of modern chemotherapy, patients with metastatic gastric cancer continue to have a dismal outcome. The 5-year relative survival for metastatic gastric cancer is $5.5 \%$ [25]. The median OS from therapy initiation until death was 13.9 months in HER2-positive patients in the ToGA trial. As mentioned above, this outcome represented a major breakthrough in modern oncology [26]. However, whether HER2-positivity is an independent prognostic marker is controversially discussed [27].

Furthermore, despite the fact that patients with peritoneal carcinomatosis with no measurable disease are known to have a significantly longer survival than that of patients with measurable disease (18.0 vs. 11.6 months), the median survival is still poor [28].

With regard to this data, the survival of our patient was exceptional. This might be due to the good performance status as well as the long treatment responses to first- and second-line therapy. Furthermore, individual treatment decisions which are made in interdisciplinary tumor boards might have contributed to this exceptional survival outcome as well. In addition, a favorable intrinsic biologic behavior of the tumor due to unknown mutations must also be considered. This underlines the importance of new prospective trials to further characterize and analyze gastric cancer cells.

\section{Further considerations}

Another important issue to discuss is the microsatellite instability (MSI). In 2017, the checkpoint inhibitor pembrolizumab was approved by the US Food and Drug Administration for the treatment of unresectable or metastatic MSI high solid tumors without any satisfactory alternative treatment options after at least one prior treatment [29]. Thus, pembrolizumab is a valid second and further line option for metastatic gastroesophageal tumors, which are MSI high. Unfortunately, the analysis of our patient's tumor showed microsatellite stability and, thus, this treatment option was not possible.

Concerning future directions, novel anti-HER2 approaches and combinations are gaining attention. A recent phase II trial showed promising results with the antibody-drug conjugate trastuzumab deruxtecan as a third and further line strategy for patients with HER2-positive advanced gastric cancer [30]. This option is expected to be practice-changing within the next couple of years.

Another potential breakthrough was recently published in June 2020 and shows that pembrolizumab combined with trastuzumab and chemotherapy has promising activity in HER2-positive metastatic esophagogastric cancer in a first-line setting [31].

\section{Conclusion}

Biomarkers such as HER2 and MSI are of high clinical interest, treatment relevant and might have prognostic impact on the OS of gastroesophageal adenocarcinoma patients. In conclusion, there is a clear first-line standard for those patients. However, second and fur- 
ther line treatment options are scarce and the clinical benefit of such strategies is under critical discussion. New treatment options are desperately needed and there are several ongoing trials, which are promising.

Funding Open access funding provided by Medical University of Vienna.

Conflict of interest H.C. Puhr has received travel support from Eli Lilly, MSD, Novartis, Pfizer and Roche. A. IlhanMutlu participated in advisory boards from MSD and Servier, received lecture honoraria from Eli Lilly and Servier, is the local PI for clinical trials sponsored by BMS and Astellas.

Open Access This article is licensed under a Creative Commons Attribution 4.0 International License, which permits use, sharing, adaptation, distribution and reproduction in any medium or format, as long as you give appropriate credit to the original author(s) and the source, provide a link to the Creative Commons licence, and indicate if changes were made. The images or other third party material in this article are included in the article's Creative Commons licence, unless indicated otherwise in a credit line to the material. If material is not included in the article's Creative Commons licence and your intended use is not permitted by statutory regulation or exceeds the permitted use, you will need to obtain permission directly from the copyright holder. To view a copy of this licence, visit http://creativecommons.org/licenses/by/4.0/.

\section{References}

1. Al-Batran S-E, Homann N, Pauligk C, Goetze TO, Meiler J, Kasper S, et al. Perioperative chemotherapy with fluorouracil plus leucovorin, oxaliplatin, and docetaxel versus fluorouracil or capecitabine plus cisplatin and epirubicin for locally advanced, resectable gastric or gastrooesophageal junction adenocarcinoma (FLOT4): a randomised, phase 2/3 trial. Lancet. 2019;393(10184):1948-57. https://doi.org/10.1016/S0140-6736(18)32557-1.

2. Tong G, LiS, Lin L, HeL, WangL, LvG, etal. Trastuzumab with FLOT regimen for the perioperative treatment of resectable HER2 + advanced gastric cancer: a retrospective study. Cancer Manag Res. 2020;12:2481-9. https://doi.org/10. 2147/CMAR.S239420.

3. Hofheinz R, Hausen GZ, Borchert K, Kretzschmar A, Ebert MP, Ettrich TJ, et al. Perioperative trastuzumab and pertuzumab in combination with FLOT versus FLOT alone for HER2 positive resectable esophagogastric adenocarcinoma: petrarca-A phase II trial of the German AIO. J Clin Oncol. 2017;35(15_suppl):TPS4133. https://doi.org/10. 1200/JCO.2017.35.15_suppl.TPS4133.

4. Group G, Paoletti X, Oba K, Burzykowski T, Michiels S, Ohashi Y, et al. Benefit of adjuvant chemotherapy for resectable gastric cancer: a meta-analysis. JAMA. 2010;303(17):1729-37. https://doi.org/10.1001/jama. 2010.534 .

5. Bang YJ, Van Cutsem E, Feyereislova A, Chung HC, Shen L, Sawaki A, et al. Trastuzumab in combination with chemotherapy versus chemotherapy alone for treatment of HER2-positive advanced gastric or gastro-oesophageal junction cancer (ToGA): a phase 3, open-label, randomised controlled trial. Lancet. 2010;376(9742):687-97. https:// doi.org/10.1016/s0140-6736(10)61121-x.

6. GongJ, Liu T, Fan Q, Bai L, BiF, QinS, etal. Optimal regimen of trastuzumabin combination with oxaliplatin/capecitabine in first-line treatment of HER2-positive advanced gastric cancer (CGOG1001): a multicenter, phase II trial. BMC Cancer. 2016;16:68. https://doi.org/10.1186/s12885-0162092-9.

7. Tabernero J, Hoff PM, Shen L, Ohtsu A, Shah MA, Cheng K, et al. Pertuzumab plus trastuzumab and chemotherapy for HER2-positive metastatic gastric or gastro-oesophageal junction cancer (JACOB): final analysis of a double-blind, randomised, placebo-controlled phase 3 study. Lancet Oncol. 2018;19(10):1372-84. https://doi.org/10.1016/ S1470-2045(18)30481-9.

8. Hecht JR, Bang YJ, Qin SK, Chung HC, Xu JM, Park JO, et al. Lapatinib in combination with capecitabine plus oxaliplatin in human epidermal growth factor receptor 2-positive advanced or metastatic gastric, esophageal, or Gastroesophageal Adenocarcinoma: TRIO-013/LOGiC-A randomized phase III trial. J Clin Oncol. 2016;34(5):443-51. https://doi.org/10.1200/jco.2015.62.6598.

9. Lopez A, Chen H-C, Harada K, Murphy MAB, Rogers JE, Thomas I, et al. Maintenance therapy with trastuzumab +/- fluoropyrimidine in non-progressive HER-2 positive advanced gastroesophageal cancer patients after first-line chemotherapy. J Clin Oncol. 2018; https://doi.org/10. 1200/JCO.2018.36.15_suppl.e16071.

10. Fu X, Zhang Y, Yang J, Qi Y, Ming Y, Sun M, et al. Efficacy and safety of trastuzumab as maintenance or palliative therapy in advanced HER2-positive gastric cancer. OTT. 2018;11:6091-100. https://doi.org/10.2147/OTT.S174138.

11. Ilhan-Mutlu A, Taghizadeh H, Beer A, Dolak W, BaSsalamah A, Schoppmann SF, et al. Correlation of trastuzumab-based treatment with clinical characteristics and prognosis in HER2-positive gastric and gastroesophageal junction cancer: a retrospective single center analysis. Cancer Biol Ther. 2018;19(3):169-74. https://doi. org/10.1080/15384047.2017.1414759.

12. Sun J, Sun Y-H, Zeng Z-C, Qin X-Y, Zeng M-S, Chen B, et al. Consideration of the role of radiotherapy for abdominal lymph node metastases in patients with recurrent gastric cancer. Int J Radiat Oncol Biol Phys. 2010;77(2):384-91. https://doi.org/10.1016/j.ijrobp.2009.05.019.

13. Kim M-S, Yoo SY, Cho CK, Yoo HJ, Yang KM, Kang JK, et al. Stereotactic body radiotherapy for isolated para-aortic lymph node recurrence after curative resection in gastric cancer. J Korean Med Sci. 2009;24(3):488-92. https:// doi. org/10.3346/jkms.2009.24.3.488.

14. MakiyamaA, Sagara K, Kawada J, Kashiwada T, HosokawaA, Horie Y, et al. A randomized phase II study of weekly paclitaxel \pm trastuzumab in patients with HER2-positive advanced gastric or gastro-esophageal junction cancer refractory to trastuzumab combined with fluoropyrimidine and platinum: WJOG7112G (T-ACT). J Clin Oncol. 2018;36(15_suppl):4011.https://doi.org/10.1200/JCO. 2018.36.15_suppl.4011.

15. Bang Y-J. A randomized, open-label, phase III study of lapatinib in combination with weekly paclitaxel versus weekly paclitaxel alone in the second-line treatment of HER2 amplified advanced gastric cancer (AGC) in Asian population: Tytan study. JClin Oncol. 2013;31(4_suppl):11. https://doi.org/10.1200/jco.2013.31.4_suppl.11.

16. Thuss-Patience PC, Shah MA, Ohtsu A, Van Cutsem E, Ajani JA, Castro $\mathrm{H}$, et al. Trastuzumab emtansine versus taxane use for previously treated HER2-positive locally advanced or metastatic gastric or gastro-oesophageal junction adenocarcinoma (GATSBY): an international randomised, open-label, adaptive, phase $2 / 3$ study. Lancet Oncol. 2017;18(5):640-53. https://doi.org/10.1016/S14702045(17)30111-0. 
17. Saeki H, Oki E, Kashiwada T, Arigami T, Makiyama A, Iwatsuki M, et al. Re-evaluation of HER2 status in patients with HER2-positive advanced or recurrent gastric cancer refractory to trastuzumab (KSCC1604). Eur J Cancer. 2018;105:41-9. https://doi.org/10.1016/j.ejca.2018.09. 024.

18. Wilke H, Muro K, Van Cutsem E, Oh S-C, Bodoky G, Shimada $Y$, et al. Ramucirumab plus paclitaxel versus placebo plus paclitaxel in patients with previously treated advanced gastric or gastro-oesophageal junction adenocarcinoma (RAINBOW): a double-blind, randomised phase 3 trial. Lancet Oncol. 2014;15(11):1224-35. https://doi.org/10. 1016/S1470-2045(14)70420-6.

19. Vita FD, Borg C, Farina G, Geva R, Carton I, Cuku H, et al. Ramucirumab and paclitaxel in patients with gastric cancer and prior trastuzumab: subgroup analysis from RAINBOW study. Future Oncol. 2019;15(23):2723-31.https://doi.org/ 10.2217/fon-2019-0243.

20. Cherny NI, Sullivan R, Dafni U, Kerst JM, Sobrero A, Zielinski C, et al. ESMO-magnitude of clinical benefit scale V.1.0 questions and answers. ESMO Open. 2016;1(5):e100. https://doi.org/10.1136/esmoopen-2016-000100.

21. Shitara K, Doi T, Dvorkin M, Mansoor W, Arkenau H-T, Prokharau A, et al. Trifluridine/tipiracil versus placebo in patients with heavily pretreated metastatic gastric cancer (TAGS): a randomised, double-blind, placebo-controlled, phase 3 trial. Lancet Oncol. 2018;19(11):1437-48. https:// doi.org/10.1016/S1470-2045(18)30739-3.

22. Alsina M, Tabernero J, Shitara K, Doi T, Dvorkin M, Mansoor W, et al. Analysis of symptoms and functional HRQoL scales in TAGS, a phase III trial of trifluridine/tipiracil (FTD/TPI) in metastatic gastric cancer (mGC). J Clin Oncol. 2019;37(15_suppl):4043.https://doi.org/10.1200/JCO. 2019.37.15_suppl.4043.

23. TohmeS, Simmons RL, TsungA. Surgery for cancer: a trigger for metastases. Cancer Res. 2017;77(7):1548-52. https:// doi.org/10.1158/0008-5472.Can-16-1536.

24. Jacquet P, Jelinek JS, Steves MA, Sugarbaker PH. Evaluation of computed tomography in patients with peritoneal carcinomatosis. Cancer. 1993;72(5):1631-6. https://doi.org/10. 1002/1097-0142(19930901)72:5.

25. National Cancer Institute. SEER cancer stat facts: stomach cancer. 2019. https://seer.cancer.gov/statfacts/html/ stomach.html. Accessed 25 Nov2019.
26. Janjigian YY, Werner D, Pauligk C, Steinmetz K, Kelsen DP, Jäger E, et al. Prognosis of metastatic gastric and gastroesophageal junction cancer by HER2 status: a European and USA International collaborative analysis. Ann Oncol. 2012;23(10):2656-62. https://doi.org/10.1093/ annonc/mds 104 .

27. Jørgensen JT, Hersom M. HER2 as a prognostic marker in gastric cancer-a systematic analysis of data from the literature. J Cancer. 2012;3:137-44. https://doi.org/10. $7150 /$ jca.4090.

28. Hong SH, Shin YR, Roh SY, Jeon EK, Song KY, Park CH, et al. Treatment outcomes of systemic chemotherapy for peritoneal carcinomatosis arising from gastric cancer with no measurable disease: retrospective analysis from a single center. Gastric Cancer. 2013;16(3):290-300. https://doi. org/10.1007/s10120-012-0182-1.

29. Le DT, Uram JN, Wang H, Bartlett BR, Kemberling H, Eyring AD, et al. PD-1 blockade in tumors with mismatchrepair deficiency. N Engl J Med. 2015;372(26):2509-20. https://doi.org/10.1056/NEJMoa1500596.

30. Shitara K, Bang Y-J, Iwasa S, Sugimoto N, Ryu M-H, Sakai D, et al. Trastuzumab deruxtecan in previously treated HER2positive gastric cancer. NEngl J Med. 2020;382(25):2419-30. https://doi.org/10.1056/NEJMoa2004413.

31. Janjigian YY, Maron SB, Chatila WK, Millang B, Chavan SS, Alterman C, et al. First-line pembrolizumab and trastuzumab in HER2-positive oesophageal, gastric, or gastro-oesophageal junction cancer: an open-label, single-arm, phase 2 trial. Lancet Oncol. 2020;21(6):821-31. https://doi.org/10.1016/S1470-2045(20)30169-8.

Publisher's Note Springer Nature remains neutral with regard to jurisdictional claims in published maps and institutional affiliations.

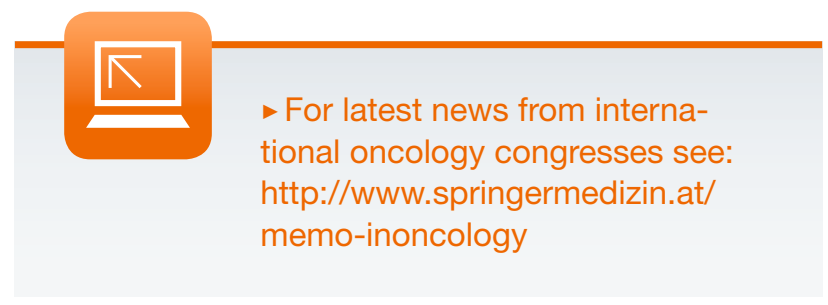


Hier steht eine Anzeige.

Springer 